\title{
Experiences de l'immigration algerienne en France et grammaires urbaines et publiques de la reconnaissance
}

Algerian immigration expeciences in France and urban and public grammars of recognition

Experiências da imigração algeriana na França e gramáticas urbanas e públicas de reconhecimento

\section{Alain Battegay}

Chercheur associé au Cnrs (LAMES-Aix en Provence UMR 7305; Centre Max Weber UMR 5283- Lyon ). Membre du Conseil Editorial de la revue "Migrations société ". Ses activités de recherches, articulant public et politique, s'inspirent d'approches pragmatiques et réflexives en sociologie et en anthropologie. Elles sont orientées vers des questions de migrations, de villes, de cultures publiques, de mémoires, en France et sur les bords du Moyen-Orient.

\section{Résumé}

Argumentant pour une méthode d’explorations situées, cet article vise à déployer des problématiques de la reconnaissance proches de l'expérience sociale, entre le quotidien et la politique, entre la reconnaissance à l'œuvre dans des interactions ordinaires et le droit à la reconnaissance. Il s'appuie sur des recherches d'inspiration socio-anthropologique portant sur la manière dont les générations issues des migrations maghrébines, notamment Algériennes, et leur installation durable à domicile travaillent les manières de faire société en France et contribuent à leur re-fabrication. Deux domaines de recherches sont ainsi revisités qui concernent 1) les approches urbaines des migrations et les grammaires des pratiques urbaines (avec une insistance plus particulière sur les " centralités immigrées »); et 2) la place de l'immigration algérienne dans la mémoire publique en France. Ces deux domaines désignent à l'attention des zones de troubles en matière de connaissance, d'ambivalences en matière de reconnaissance marquées par des déficits, des différentiels, des registres dissociés dans l'expérience des populations d’origine Algérienne vivant en France, même si elles sont de nationalité française, qui inquiètent les conceptions des sociabilités d'interconnaissance en signalant la vulnérabilité des dispositifs d'inter-reconnaissance. 
Mots clés: Connaissance, Reconnaissance, Inter-Reconnaissance, Migrations Algériennes En France, Explorations Situées, Approches Urbaines, Grammaires Des Pratiques Urbaines, «Centralités Immigrées », Mémoire Publique, Troubles, Déficit, Différentiel, Vulnérabilité.

\section{Abstract}

Arguing for a method of situated explorations, this article aims to deploy problematics of recognition close to the social experience, between daily life and politics, between recognition at work in ordinary interactions and the right to recognition. It is based on researches of socio-anthropological inspiration on how the generations resulting from Maghreb migrations, especially Algerian, and their durable installation at home, work the ways to make society in France and contribute to their re-fabrication. Two fields of research are thus revisited regarding 1) urban approaches to migration and grammars of urban practices (with a particular emphasis on "immigrant centralities"); and 2) the place of Algerian immigration in the public memory in France. These two domains refer to areas of unrest in terms of knowledge, of ambivalences with regard to recognition marked by deficits, differentials, dissociated registers in the experience of the Algerian population living in France, even if they have a French nationality, who concern conceptions of sociability of inter-knowledge by signaling the vulnerability of inter-recognition devices.

Keywords: Knowledge, Recognition, Inter-Recognition, Algerian Migrations in France, Situated Explorations, Urban Approaches, Grammars of Urban Practices, "Immigrant Centralities", Public Memory, Troubles, Deficit, Differential, Vulnerability.

\section{Resumo}

Argumentando por um método de explorações situadas, este artigo tem por objetivo implantar problemáticas de reconhecimento próximas da experiência social, entre o cotidiano e a política, entre o reconhecimento no trabalho em interações comuns e o direito ao reconhecimento. Baseia-se em pesquisas de inspiração socio-antropológica sobre como as gerações resultantes das migrações magrebinas, argelinas em particular, e sua instalação duradoura 
em casa, trabalham as formas de fazer a sociedade na França e sua refabricação. Duas áreas de pesquisa são, portanto, revisitadas: 1) abordagens urbanas de migração e gramáticas de práticas urbanas (com insistência particular nas "centralidades imigrantes"); e 2) o lugar da imigração argelina na memória pública na França. Esses dois campos referem-se a áreas problemáticas em termos de conhecimento, de ambivalências em relação ao reconhecimento marcadas por déficits, diferenciais, registros dissociados na experiência de populações de origem argelina que vivem na França, mesmo com nacionalidade francesa, que inquietam as concepções de sociabilidade do conhecimento mútuo, sinalizando a vulnerabilidade dos dispositivos de reconhecimento mútuo.

Palavras-chave: Conhecimento, Reconhecimento, Inter-Reconhecimento, Migrações Argelinas na França, Explorações Situadas, Abordagens Urbanas, Gramáticas de Práticas Urbanas, “Centralidades Imigrantes”, Memória Pública, Problemas, Déficit, Diferencial, Vulnerabilidade.

Cet article propose une réflexion sur le thème de la reconnaissance à partir de recherches d'inspiration socio-anthropologique portant initialement sur l'expérience en France des générations issues des migrations maghrébines et notamment Algérienne, depuis la fin années 70/début des années 1980, leur accès à l'espace public et les processus ambivalents de reconnaissance impliqués (BATTEGAY, 1985). Ces recherches ont, dans un premier temps, suivi des chemins d'accès à la ville et à la vie publique de ces générations largement ignorées jusqu'alors, identifiés comme des délinquants de type maghrébin à la rubrique des faits divers de la presse locale reprenant les catégories policières, - et comme des enfants illégitimes (SAYAD, 1979) et des êtres en négatifs (BEKKAL, 1981) par la littérature sociologique de l'époque. Les enfants des familles immigrées, et particulièrement les jeunes issus de l'immigration algérienne, sont progressivement devenus une des figures des renouvellements de la recherche sur les banlieues et l'immigration en France en même temps qu'ils s'imposaient comme figures d'actualité. Leur émergence sur la scène publique a attiré l'attention sur les transformations d'une immigration de travail en une immigration familiale et de peuplement dans un contexte de crise de l'emploi 
qui prive l'immigration de ses justifications économiques, ainsi que sur les processus de ségrégation/regroupement résidentiels de familles immigrées, notamment du Maghreb, dans les grands ensembles périphériques devenus lieux de cohabitations interethniques.

Nombre de ces études sociologiques et anthropologiques ont été conçues comme portant sur les processus et les conditions d'intégration en France de populations dont la présence était perçue comme temporaire, et qui est progressivement apparue, au cours de ces années, comme durable. Très fortement marquées par la culture politique française et la doctrine républicaine, elles ont repris à leur compte cette perspective de l'intégration (DEWITTE, 1999) ${ }^{1}$ même si, récemment, la notion de société inclusive (TUOT, 2013) déplace en partie l’orientation des regards: elles ont d'avantage décrypté les épreuves d'intégration comme des épreuves interrogeant les capacités d'adaptation des immigrés à la vie en France, plutôt qu'interrogeant les ouvertures, fermetures et replis de la société française. Quoiqu'il en soit, toutes ces études confirment l'hypothèse que l'installation durable à domicile de populations issues de l'immigration maghrébine constitue un événement dont le poids démographique et les résonnances sociales, culturelles, politiques n'ont pas fini de travailler les manières de faire société en France.

Pour approcher les portées de cet événement, nous proposons de centrer l'attention sur les figures de reconnaissance inédites qui émergent dans les suites de cet événement et sur la re-fabrication des grammaires d'un vivreensemble en France auxquelles il conduit. Ces figures s'inscrivent dans des historicités variables. Les nouveaux visages publics des banlieues émergent dans des histoires de longue durée, celle des banlieues ouvrières et des quartiers d'habitat social, celle de l'histoire coloniale et post coloniale entre la France et l'Algérie; leur émergence même est désormais affaire de moyenne durée à l'échelle de générations démographiques, de mouvements récurrents de

Ce document témoigne de la prégnance du couple immigration/intégration dans la recherche française, et de la centralité accordée aux relations entre Français et immigrés dans le champ des études sur les relations interethniques: les relations entre différentes immigrations sont très peu prises en compte si ce n'est dans le cadre d'études contrastives ou comparatives. 
révolte ou de rébellion, de la montée en visibilité de l' « ethnicité » en France comme catégorie disputée de perception et de traitement, et comme principe organisationnel de composition résidentielle et d'agencements urbains. Dans les trames continuées d'actualité médiatique, la figure du musulman, de longue historicité en France et en Europe, devient, à partir des années 1990 et plus fortement encore ces dernières années, omniprésente - une catégorisation religieuse fonctionnant presque comme une catégorie « ethnique » à l'échelle de la France et de l'Europe qui font désormais du contrôle migratoire et de la lutte contre le terrorisme un sujet politique et moral qui rend suspectes les populations musulmanes issues des migrations.

\section{PENSER LA RECONNAISSANCE A PARTIR D'EPREUVES EN SOCIETÉ}

Dans la pensée, ces figures, identifiées par des explorations anthropologiques, sociologiques, historiques, conduisent à recourir et à mettre à l’épreuve la notion de la reconnaissance, abondamment utilisée dans des approches de sciences politiques et de sociologie politique. Telle était une perspective de l'exercice proposé dans « La reconnaissance à l'épreuve » (PAYET; BATTEGAT, 2008) qui visait à tester les capacités des théories et des problématiques de la reconnaissance à rendre intelligible les épreuves de la vie institutionnelle, publique, urbaine, morale des faire-société contemporains. Cela avait alors conduit à déplier une sorte d'analytique de la reconnaissance en identifiant, sans prétention d'exhaustivité, différentes formes de reconnaissance situées et à l’œuvre: les manières dont la reconnaissance est assignée dans les mondes de la relation institutionnelle, conditionnée par un travail de cadrage identitaire, éprouvée dans des expériences de la vulnérabilité... Tout en interrogeant, dans des perspectives internationales et de longue durée des dispositifs de traitement et de reconnaissance de l'altérité et de l'individu dans des modernités différenciées. Cette ébauche d'une analytique de la reconnaissance avait moins comme ambition de contribuer à l'architecture 
théorique d'un paradigme ou d'une théorie de la reconnaissance, qu'à déployer des problématiques de la reconnaissance proches de l'expérience sociale, entre quotidien et politique, entre reconnaissance à l'oeuvre dans des interactions ordinaires et droit à la reconnaissance. Elle accordait ainsi la priorité à l'approche de situations dans lesquelles les enjeux de reconnaissance sont brouillés, complexes, jouant simultanément sur plusieurs registres et sur plusieurs scènes.

Trois indications peuvent être retenues de ce moment de recherche et de ces travaux:

- Une première indication, d’ordre théorique, concerne les expressions académiques et les traditions de recherches mobilisées par les approches de philosophie politique de la reconnaissance. Elle consiste à souligner l'importance d'une vaste tradition de pensée et de recherches qui traitent de la considération (BORDREUIL, 2008), représentée par les travaux de Durkheim, Mauss, Gurvitch - tradition de recherches de la sociologie « classique » en France - mais aussi de Simmel et Goffman. Ces travaux réfléchissent au don, à la dette, et aussi au respect, à l'honneur, à la face: à des économies et des grammaires morales que les philosophies politiques de la reconnaissance visitent peu, se souciant plutôt de l'application du principe dégale dignité de tous (TOTO; PENIGAUD; RENAULT, 2017);

- La deuxième indication, méthodologique, plaide pour des approches des processus de reconnaissance à léchelle des mœurs et des manières de faire société. Il s'agit ici de rester «dans la caverne » (WALZER, 1987), proche de l'ordre de l'interaction autant que de l'ordre social, l'un ne se déduisant pas de l’autre, ce qui ne les empêchent pas dêtre liés dans une sorte de couplage flou commel'a signalé Goffman (1988). Ce couplage flou justifie d'une certaine manière des explorations situées de la reconnaissance, en les orientant selon une méthodologie qui approche les situations contemporaines à la fois « du dedans » (dans laction en train de s’accomplir) et « d’en haut », à léchelle de régularités 
impersonnelles et de normes de conjonction qui ouvre des fenêtres de compréhension sur les manières de faire société;

- La troisième indication, enfin, concerne les liens entre scientifique et politique. Loin de soutenir une posture qui « monte en grandeur politique » des demandes de reconnaissance, il s'agit de soutenir que l'intérêt scientifique de connaissance des épreuves de reconnaissance est politique autant que documentaire par la contribution qu'elle peut apporter à une intelligibilité publique est politique - ce qui est une manière de considérer, dans le travail du sociologue, les rapports entre politique et scientifique.

Pour revenir à notre objet, la manière dont les générations issues des migrations maghrébines et notamment Algériennes et leur installation durable à domicile travaillent la société française et contribuent à sa re-fabrication, cette méthode d'explorations situées conduit à revisiter les problématiques de la reconnaissance en tentant une double inflexion:

- La première consiste à urbaniser les théories de la reconnaissance développées en philosophie politique, et à urbaniser la notion de grammaire, en la détachant en partie des répertoires argumentatifs pour insister sur des morphologies, des arrangements urbains et des parcours socio-spatiaux qui dessinent des sortes de syntaxes urbaines du vivre-ensemble, composées d'évitements et de mises à distance autant que de scènes de croisement plus ou moins problématiques. A léchelle des ordres urbains, les approches des situations des populations maghrébines en France, font apparaitre des pratiques en demi teinte de reconnaissance des embarras et des régimes ambivalents d'inter-reconnaissance;

- La seconde consiste à explorer la place faite à l'immigration Algérienne dans la mémoire publique en France, et particulièrement dans les mouvements de redéploiement de la mémoire publique nationale, des gestes présidentiels à la figuration sur des scènes muséales et 
mémorielles, des musées et des lieux de mémoire aux animations locales, des commémorations publiques aux noms des rues et aux conflits dans la toponymie urbaine. Ce contexte incite à explorer des rapports au passé mobilisés dans les manières contemporaines de faire société, entre mémoires à l’œuvre et paysages mémoriels en interaction. Là aussi se lisent des régimes et des malaises de reconnaissance, qui coexistent et interfèrent, dans la mémoire publique nationale.

Dans la pensée, ces malaises dans la reconnaissance sont simultanément des malaises dans la connaissance. Le terme d'ethnicité - et a fortiori d'ethnicité postcoloniale - fait en effet partie du malaise dans un paysage intellectuel et scientifique qui, en France, a longtemps privilégié la question sociale sur la question ethnique (NICOLAS, 1973), et d'autant plus que le terme d'ethnie est entaché de son utilisation coloniale et antisémite. Mais le terme d'ethnicité ne fait pas ici référence à un stock de traits culturels distinctifs et de spécificités importées ou transplantées par les immigrés dans leurs déplacements et leurs valises. Pour le dire d'une formule: «Il était kabyle en Algérie, berbère au Maroc et il se retrouve arabe en France ». Cette ethnicité-là - qui s'entend dans les usages vernaculaires des termes «arabes» ou «noirs »- ne se déduit pas d'une ethnicité importée. Elle signale et désigne des manières publiquement affirmées de faire société. Une telle affirmation ne conduit pas à ethniciser ou à islamiser la question sociale: elle se réfère à une conception non-essentialiste de l'ethnicité (MARTINELLO, 1995), dans un contexte où ces altérités ne peuvent pas être pensées comme des produits importés, «extérieurs» à la société qui les qualifie et leur donne sens. Les populations d'origine ou issues des migrations maghrébines vivant en France sont souvent ainsi désignées par commodité de langage (il en sera ainsi dans la suite de cet article) mais de manière en partie inadéquate. Elles sont désormais souvent intégrées dans la nationalité française, ont statut de citoyens mais restent perçues comme des étrangers de l'intérieur. Pour la plupart, elles ne relèvent pas du statut juridique et administratif d'étranger même si dans une même famille 
peuvent se retrouver des membres français et d'autres de nationalité étrangère (algériens, marocains, tunisiens) et que la part des 'doubles nationaux', de fait et non de droit, n'est pas négligeable. Le malaise dans la connaissance en France de l'ethnicité comme phénomène relationnel et organisationnel généré dans les mouvements des coexistences est une première épreuve de reconnaissance, non seulement de la légitimité de présence de ces populations mais de manières contemporaines de faire société. Cette épreuve mérite pourtant d'être explorée par le milieu, dans les interactions de la ville milieu et de la vie publique qui la coproduise (BOUBEKER, 2003).

\section{LA RECONNAISSANCE DES GRAMMAIRES URBAINES DE L'ETHNICITE EN FRANCE}

Depuis le début des années 80, les «banlieues » et «l'immigration » sont en France, à l'agenda de préoccupations médiatiques, politiques, scientifiques et d'évènements "de société » et d'actualité urbaine. Les termes de banlieues et d'immigration sont d'ailleurs en partie inadéquats au regard des situations et des populations impliquées. Toutes les banlieues et toutes les immigrations ne sont pas concernées, - seulement une partie des banlieues qui se composent d'une grande part de logement social, et seulement les immigrations et les populations issues de l'immigration du Sud de la Méditerranée, venant principalement du monde Arabe et d'Afrique noire, alors même qu'une partie de ces populations sont désormais en grande partie de nationalité française.

Dans les années 80, les rapports villes-banlieues ont été compris en France comme des métaphores d'une société fonctionnant désormais sur le couple inclus/exclus et non plus seulement sur des rapports de classe (d'intégration par la lutte). Ces métaphores s'appuient sur des diagnostics qu'on pourrait qualifier de morphologie urbaine, au sens que donne Halbwachs (1950) à la notion de morphologie en liant formes spatiales et formes sociales. Elles méritent d’être dépliées selon un double 
regard: les villes vues d'en haut, dans leurs compositions résidentielles et sociodémographiques, et les villes vues du dedans, à l'échelle des citadins et des manières de la vivre.

\section{LA VILLE VUE D'EN HAUT: COMPOSITIONS HABITANTES}

Au début des années 80, les analyses qui se sont développées concernant les processus de ségrégation et de concentration résidentielles de populations défavorisées ont dû tenir compte des populations "immigrées », arabes, maghrébines sans savoir comment les nommer, ni comment les compter. Le terme de ghetto a fait alors controverse, comme celui de seuil de tolérance. La doctrine qui s'est progressivement élaborée a eu tendance à refuser la pertinence de ce terme de ghetto au regard des situations observables, identifiées comme des "poches de surconcentration des précarités ». Ce vocabulaire a alors fait passer au second plan les caractères d'ethnicité de ces relégations et de ces territoires, sortes d'épines dans le pied des conceptions républicaines de la gestion urbaine. Le malaise dans la reconnaissance publique de l'ethnicité en France limite la connaissance de l'ethnicité comme principe d'allocation voire d'organisation de l'espace, puisque les catégories ethniques (avec des critères comme arabes ou noirs) sont interdites dans les recensements français, conformément à la philosophie et la doctrine républicaine qui prévaut. L'absence de ces catégories produit une invisibilité statistique de ces populations et plus généralement des dimensions d'ethnicité dans la société française (SIMON; STAVO-DEBAUGE, 2004), que ce soit dans les répartitions de populations à l'échelle des agglomérations, ou dans l'accès à des ressources pour des configurations de populations dont le nom reste incertain: immigrés, issus des migrations, musulmans, arabes. Dans la plupart des analyses urbaines, la question de l'ethnicité n'apparaît qu'en tant qu'épreuve supplémentaire 
et mal dimensionnée de la réduction des inégalités sociales, qui, elles sont amplement documentées.

Dans le même temps, la concentration des familles immigrées d'Afrique subsaharienne du Maghreb et des générations dans les quartiers et les morceaux de ville périphériques des grandes agglomérations, ont continué à marquer les compositions résidentielles des villes françaises, faisant de l'ethnicité un principe d'allocation de l'espace et une ligne durable de qualification des territoires. Les «banlieues » sont alors devenues un terme métonymique évoquant les fractures socio-spatiales des villes contemporaines, l'expression de «ville à deux vitesses » devenant une expression ordinaire pour signifier de nouvelles formes de conflictualités sociales, difficilement réductibles à celles que la France avait l'habitude de percevoir dans sa propre histoire et dans son actualité.

A partir des années 1990, de nouveaux diagnostics ont prévalu, donnant à lire, non des « villes à deux vitesses », mais des « villes à plusieurs vitesses ». Le couple banlieue/ville a semblé alors insuffisant pour décrire l'étalement de l'urbain: l'attention a été attirée sur le péri-urbain et une troisième vitesse, celle des classes moyennes qui sortent des villes et des banlieues pour habiter là où les villes semblent se vivre à la carte. La question des fragmentations urbaines se poserait ainsi à l'échelle d’agglomérations et de conurbations plutôt que dans le couple ville-banlieue. Létalement des villes devenant agglomérations et aire urbaines d'une part, et de nouvelles formes de les habiter et de les vivre d'autre part ont alors incité les observateurs à recourir aux métaphores de la ville archipel, de la ville éclatée, de la ville fragmentée pour interroger l'émergence de nouvelles formes de centralités ainsi que les nouveaux rôles des villes centres qui se gentrifient. Dans ce contexte, le couple ville/banlieue a perdu de sa pertinence. Les frontières urbaines et sociales qu'il entendait désigner se sont à la fois diluées dans un tableau plus général en faisant apparaître des territoires restreints et identifiés de concentration des précarités mais étendus au-delà de la géographie des quartiers prioritaires qui avait été tracée. Pour autant, les qualifications socio-ethniques de ces espaces et des populations qui y résident se sont maintenues, dans les 
représentations et aussi dans les compositions habitantes de ces morceaux d'agglomérations et d'aires urbaines - même si le couple ville-banlieue est insuffisant pour les décrire (BATTEGAY, 1992).

\section{LA VILLE VUE DU DEDANS ET LES PRATIQUES D'APPROVISIONNEMENT DES MUSULMANS EN FRANCE}

Cette connaissance des compositions habitantes est, bien entendu, loin de suffire à l'analyse des manières de vivre les villes. Les habitants de ces quartiers de banlieue, parmi lesquels de nombreux migrants, fréquentent aussi d'autres espaces urbains que ceux de leurs quartiers de résidence. Ce sont aussi des consommateurs, qui s'approvisionnent entre commerces de proximité, marchés urbains de plein air, grands centres de distribution et aussi espaces "spécifiques», « commerces ethniques» et "places commerçantes immigrées ». L'organisation sociale et urbaine de l'activité de distribution aussi une épreuve de reconnaissance publique de styles de consommation minoritaires qui désignent à l'attention des formes urbaines originales qui ont été explorées par des démarches d'anthropologie urbaine.

\section{« COMMERCES ETHNIQUES »...}

Au cours des années 2000, des recherches urbaines se sont en effet développées sur les "commerces ethniques", qui marquent les paysages et les compositions urbaines autant que les manières de vivre les villes en France. Dans ses usages par les sciences sociales, l'expression « commerce ethnique», qui s'inspire de la notion d'ethnic business, se distingue de la notion de « commerce étranger ». Elle ne désigne pas des commerces développés par certains groupes de populations ou certaines « ethnies » disposant de cultures commerçantes ou entrepreneuriales particulières ou de stocks de 
traits culturels distinctifs hérités. Bien au contraire: le terme " commerce ethnique » relève de démarches de recherches qui ont pensé l'ethnicité contre l'ethnique (MARTINELLO, 1995), c'est-à-dire non comme une culture importée ou transplantée mais comme une production indigène de la société dite d'accueil qui donne sens à des différences comme mode de classement, de désignation, de qualification. En ce sens, le terme " commerce ethnique » est un terme par défaut, qui, certes, a l'immense inconvénient de sembler accréditer l'idée que " certains sont plus ethniques que d'autres », mais qui a l'avantage de rendre sensible le caractère caduque du terme étranger au regard des complexités contemporaines, et de prendre acte de la montée de l'ethnicité comme manière de faire société.

Plusieurs recherches ont ainsi porté sur les commerces " ethniques » dans les villes en France et leur multiplication, en mettant en évidence leur importance dans les recompositions actuelles du commerce urbain. Elles ont montré qu'ils se sont installés dans des failles du système commercial, dans des zones de fragilité crées par la crise du petit commerce urbain: la réorganisation des circuits de distribution à l'échelle des villes fournissent des opportunités urbaines et économiques d'installation à ces commerces ethniques dont les façades commerçantes, les vitrines, font sens, non sur le registre exclusif des expressions identitaires, mais comme dispositifs de signalement et de captation de clientèles ajustés à des contextes urbains (RAULIN, 2000). Leurs mises en scène et leurs façades (banalisées, exotiques, “communautaires") (MA MUNG, 1999) se modulent différemment selon les contextes urbains. En s'adressant à plusieurs types de clientèles, en adaptant leurs produits et leur services, en s'intégrant dans le paysage urbain par une mise en scène modulée selon les situations, les petits épiciers « étrangers » font figure de repreneurs d'unités commerciales de distribution menacées de disparaître. S’intégrant en effet aux habitudes locales de consommation et d'approvisionnement alimentaire de clientèles potentielles sans faire des clientèles « communautaires » leur seule cible commerciale, ils se banalisent tout en développant parfois leur aspect exotique, se rapprochent de leurs clientèles par des services appropriés (jours et heures d'ouvertures, livraisons à 
domicile...). L'existence d'une demande « communautaire » non-satisfaite n'est pas le ressort essentiel de leurs dynamiques de développement qui reposent sur la conquête de clientèles de proximité, ou de clientèles qui diversifient leur consommation alimentaire en intégrant une part d'exotisme.

\section{... ET « PLACES COMMERÇANTES IMMIGREES »}

Du point de vue des compositions et des agencements urbains, de la ville vue d'en haut, l'attention a aussi été portée sur ce qu'il est convenu d’appeler " centralités immigrées », centralités minoritaires (Belzunce à Marseille, la Goutte d'Or (TOUBON; MESSAMAH, 1991), Barbès, le XIII (GUILLON; TABOADA-LEONETTI, 1986; RAULIN, 1988), Belleville à Paris, La Place du Pont à Lyon) qui sont apparues en France dans les plis des évolutions urbaines depuis plus d'un demi-siècle et qui ont été un des hauts-lieux de la montée et de l'affirmation de l'ethnicité dans les villes françaises (BATTEGAY, 2008). Ces centralités commerçantes immigrées de centre-ville ont des histoires, des positionnements urbains, des modes de formation, de recomposition et de redéploiement différents, particuliers à chaque ville et qui s'articulent à des configurations et des histoires migratoires singulières. Mais leur observation a aussi fait apparaître des caractéristiques communes.

Ces recherches ont montré d'abord que les commerces de ces centralités font l'objet d'une double évaluation (TARRIUS, 1988 e 1990): d'un côté ils sont considérés comme des espaces dont la valeur foncière est dévalorisée lorsqu'ils sont échangés sur le marché local des emplacements commerciaux; mais simultanément ces commerces sont hautement valorisés lorsqu’ils font lobjet de transactions sur un marché «immigré».

Cette double valeur a incité à des explorations des «places commerçantes immigrées» exerçant des fonctions de centralité dans l'approvisionnement de populations en situation migratoires résidant dans les régions concernées, leur attractivité s'exerçant bien au-delà. 


\section{LA « PLACE DU PONT » A LYON, ESPACES ET MONDES}

Dans une recherche menée sur la Place du Pont, centralité commerçante immigrée à tonalité maghrébine de l'agglomération lyonnaise, le constat s'impose en effet d'un décalage entre d'un côté les autorités urbaines qui traitent ce morceau de ville comme un espace résiduel, plus ou moins dégradé, promis à restructuration pendant plusieurs années à travers de nombreuses opérations urbaines, et de l'autre côté son attractivité maintenue, sa valorisation continuée dans les parcours des mondes de l'immigration maghrébine, à l’échelle de la ville, l'agglomération, de l'aire urbaine. La Place du Pont est ici en même temps, morceau de ville résiduel au destin incertain, - et petit carrefour urbain des migrations maghrébines, espace de ressources attractif pour des populations dont les manières de vivre en migration se diversifient et se recomposent.

Les différentiels de valorisation, de connaissance et de reconnaissance sont ici manifestes.

Ces différentiels sont particulièrement sensibles au moment du Ramadan qui donne à la Place du Pont un rôle original à léchelle de la ville et de l'agglomération. C'est la seule scène urbaine où les pratiques liées à cet événement sont mises en scène collectivement et commercialement à l'échelle d'un morceau de ville et publiquement affirmées. Au moment du Ramadan, la scénographie commerçante de la Place se modifie: un petit marché de plein air dédié s'organise à la limite de l'informalité, les pâtisseries se multiplient, les boucheries soignent l'accueil de leurs clients qui viennent ici s'approvisionner en ambiance et en relations autant qu'en produits et en services. Les clients sont aussi des publics prenant part à cette scène urbaine du Ramadan: leur fréquentation, à ce moment de la vie collective, de cette scène urbaine est aussi participation aux rythmes collectifs et aux ritualités minoritaires du monde de l'immigration maghrébine et musulmane. L'activité de la Place du Pont au moment du Ramadan met en scène et en oeuvre une «norme de considération appropriée » (WALZER, 1997), élément fort de son attractivité auprès des populations musulmanes de l'agglomération au regard desquels elle apparait comme une ressource urbaine de reconnaissance et d'inter-reconnaissance. 
Se manifestent ici non seulement des différentiels de reconnaissance, mais aussi des dédoublements de registres d'inter-reconnaissance, dessinant des frontières urbaines du vivre ensemble, espaces et mondes.

\section{LA PLACE DES « HOMMES DEBOUT »}

La Place du Pont est ainsi, au regard de la presse et des autorités locales, une «séquence urbaine compacte et ambiguë», un quartier de deal, un quartier louche, notamment en raison de la présence d"” hommes debout " qui se regroupent, à une des entrées du quartier, au pied d'un bâtiment moderne nouvellement construit et en dépit du mobilier urbain destiné à provoquer l'inconfort des rencontres. Ces regroupements d' « hommes debout » reprennent une ancienne tradition de lieu de sociabilités, de rencontres, d'informations et de marché informel datant de plus d'un quart de siècle, - un ensemble de pratiques articulées et difficilement dissociables.

C'est un monde d'hommes: un petit marché aux puces, informel, où s'étalent des objets disparates et sans grande valeur (vêtements d'occasion, montres, ustensiles de maison), de la revente de matériel (portables..) dont la provenance est incertaine, des pratiques de change informel (un de nos interlocutrices plaisantant en parlant de ce regroupement comme de "Wall Street ", " si tu veux savoir le cours de l'or et du dollar, il n'y a pas mieux pour se renseigner "), et certains mentionnent qu'ils trouvent là des informations intéressantes pour des bonnes affaires: lots à acheter, voitures d'occasion, fonds de commerce. C'est aussi un lieu de deal et de captation de clientèles venant chercher de la drogue.

Lorganisation sociale de cet espace de regroupement est ainsi plus complexe qu'il n'y paraît: des observations montrent comment des anciens de l'immigration en ont fait un lieu d'activités et de présence relativement régulière alors que les points de rencontres qu'ils fréquentaient à proximité de leurs lieux d'habitation se sont défaits, les anciens n'étant plus là, les lieux eux-mêmes ayant changé, ou les cafés ayant disparus; elles montrent également 
la fonction-repère de ces regroupements pour ceux qui viennent d'arriver, parfois dans l'urgence ou dans des situations de clandestinité, et qui sont à la recherche de relations et de ressources, de travail, de papiers; elles montrent encore comment des populations de l'Est européen, connues sous le nom de « roumains » s'y retrouvent et utilisent parfois ces moments et ces espaces de regroupement comme une occasion de marché, des fourgonnettes apportant sur les lieux des vêtements et d'autres objets destinés à la vente. Ces différents usages qui participent à la permanence de cet espace temps de regroupement en font aussi un lieu d'intrigue qui alimente des tensions et des rumeurs: tel commerçant riverain lors d'une réunion publique a porté haut et fort la plainte de l'insécurité et de la gêne provoquée par les hommes debout; d'autres tentent de dissocier les anciens de l'immigration qui viennent s'occuper et qui font partie de la Place même s'ils n'y habitent pas, des étrangers qui viennent d'ailleurs et font de ces regroupements des lieux de deals qui nuisent à tout le quartier et à son image; des habitants soulignent le climat de tension qui prévaut à certains moments, en faisant référence autant à la présence policière qui vient faire des contrôles, de plus en plus fréquents et visibles au moment des campagnes électorales, qu’à des remarques et des gestes qui les insécurisent sans les viser. Pour ceux qui fréquentent le quartier occasionnellement, les avis sont partagés: certains trouvent que les "hommes debout" font tâche et qu'ils nuisent à l'image du quartier, d’autres sont positivement intrigués par l'animation du lieu alors que la ville est déserte le dimanche après-midi, d'autres encore remarquent, en le déplorant ou en s'en félicitant, que cette présence marque l'échec de l'opération de rénovation-nettoyage du quartier, et certains remarquent avec une certaine admiration la permanence de ce regroupement, la force des habitudes et de la ritualisation de cet espace de rencontres, qui persiste en dépit de toutes les tentatives, urbanistiques et policières qui visent à déloger les “ hommes debout ". Et si les avis sont partagés sur l'appréciation de la présence des "hommes debout", les lignes de partage de ces appréciations suivent des frontières sinueuses qui ne se confondent pas avec le fait d'habiter le quartier, d'y tenir commerce ou de venir s'y approvisionner, avec le fait d'être d'origine maghrébine ou de ne pas l'être, avec le fait d'être une femme ou un 
homme, un jeune ou un vieux. Mais à la conjonction de ces avis, "les hommes debout" s'imposent comme une des figures de la Place, ils font partie du paysage et des mondes de la Place, ils en forment un des mondes, une des images, un des emblèmes, une des ambiances, manifestant là que la frontière est poreuse entre l'illégal et l'illégitime, entre l'interconnaissance et l'inter-reconnaissance, entre l’accessibilité réservée et la fréquentation publique.

\section{L'ATTRACTIVITE DES OFFRES COMMERÇANTES DE LA PLACE DU PONT}

Les explorations que nous avons conduit sur l'attractivité continuée de la Place du Pont, nous ont aussi amener à identifier, à l’ouvre, d'autres épreuves de reconnaissance, d'interconnaissance, d'inter-reconnaissance impliquées dans le renouvellement permanent de ses offres de services et de produits. Lattractivité continuée de la Place témoigne en effet de la pertinence de ses offres au regard de populations essentiellement maghrébines qui en font des usages "intercalaires", venant chercher ici ce qu'elles ne trouvent pas ailleurs (ambiance, relations, produits, services) alors même qu'elles fréquentent d'autres centres d'approvisionnement et qu'elles commencent à être prises en considération par les grands réseaux de distribution (notamment dans l'alimentaire).

La Place du Pont qui ne peut plus compter sur les seuls bénéfices de niche pour attirer des clientèles qui ne sont pas de proximité et qui ne sont plus captives, dont les univers d'approvisionnement se diversifient, recompose continuellement son offre. Elle devient ainsi une vitrine où se mettent à l'épreuve de nouveaux produits et services, et elle est conduite à renégocier, dans ses principes de fonctionnement, les liens entre normes d'appartenance et normes marchandes, entre normes de confiance et normes de qualité, entre valeur traditionnelle et valeur d'innovation.

Au tournant des années 2000, l'analyse des spécialités commerçantes et des types de service concentrés sur ce morceau de ville montre que les services à la mobilité sont ici particulièrement denses: agences de voyages, services de 
cars, bureaux d'import-export d'automobiles, agences de services téléphoniques à la carte. Cette concentration d'offres de biens et de services en matière de déplacements s'articule à d'autres offres de biens et services dont la valeur est liée à des styles de consommation, des types de calendriers, des rituels spécifiques: boutiques liées aux mariages, aux fêtes, aux cérémonies (robes, bijouteries, bazar, et plus récemment agences d'organisation de mariages proposant à la carte ou au menu un ensemble de services: réservation de salles, location de robes de mariées, d'accessoires, de tenues vestimentaires, traiteur, et jusqu'à la location de services de la $\mathrm{Negafa}^{2}$ et au tatouage au henné des mains de la mariée); pompes funèbres musulmanes qui proposent des formules diversifiées d'enterrement, sur place dans un carré musulman ou avec rapatriement du corps en Algérie, alors que le rapatriement des corps était auparavant l'affaire des familles, des solidarités d'originaires en migration, de l'Amicale des Algériens en Europe; librairies musulmanes nouvellement implantées qui proposent autant des livres et des cassettes, vidéo et audio, en français et en arabe, en rapport avec l'Islam -elles en sont parfois l'éditeur-, que des objets liés à l'exercice du culte, ou des vêtements affichant une singularité musulmane; boucheries " halal ", qui proposent aussi de nombreux produits de charcuterie, le label « halal» étant devenu depuis le début des années 90, en France et à Lyon en particulier, un des procédés majeurs pour convaincre le consommateur de l'authenticité halal et un enjeu fort de crédibilité pour des réseaux musulmans qui investissent le domaine. En lien avec des bouchers, ils donnant à leurs entreprises commerciales l'aspect d'entreprises morales, favorisant l'accès des musulmans à des produits et services leur permettant de pratiquer leur foi. C'est en sens qu'ils font de la Place du Pont une vitrine, un espace d'expérimentation de leurs produits et services, d'épreuve de leur réputation et de leur crédibilité.

Approcher des tels morceaux de ville, c'est ainsi d'abord documenter cette superposition dans les mêmes espaces de syntaxes d'usages qui s'interconnectent et se dissocient, en configurant des territoires aux significations ambivalentes.

«Négafa » désigne une femme chargée de préparer la mariée, de la maquiller et de l’aider lors du changement des sept tenues traditionnelles. Comme le tatouage au henné des mains de la mariée, ce sont là des actes qui sont, dans les habitudes algériennes, habituellement assurés par les membres de la famille, qui considèrent qu'ils sont trop chargés de signification pour être confiés à un étranger. 
C'est aussi prendre acte de leurs déficits et différentiels de reconnaissance, des doubles lectures qui les valorisent et les dévalorisent dans des mouvements concomitants, et des usages simultanés. Ces espaces dévalorisés sont aussi centralités immigrées qui donnent ainsi à lire des scènes et moments qui sont ceux de carrefours urbains et marchands des migrations: ils forment moins des quartiers que des localités marchandes participant de villes « immigrées » enchâssées dans les villes.

Cela incite à développer des conceptions de la reconnaissance qui permettent de rendre compte de ces épreuves à l'échelle du citadin et des mœurs urbaines, marquées par des ajustements autant que par des conflits, par des évitements et des côtoiements autant que par des rencontres; à revisiter les rapports entre ségrégation et mixités, cohabitations urbaines et voisinages, contiguités et pluralisme (JOSEPH, 2002); à en déplier les syntaxes socio-spatiales; à être attentif aux usages des lieux dans des parcours et pas seulement aux images des lieux dans les représentations locales. De telles recherches parient sur l'intérêt politique et pas seulement documentaire de connaissance de ces situations dans lesquelles se noue la complexité des processus de reconnaissance, voire la dissociation des registres d'inter-reconnaissance.

\section{L'IMMIGRATION ALGERIENNE DANS LES GRAMMAIRES DE LA MEMOIRE PUBLIQUE EN FRANCE}

Cette complexité des processus de reconnaissance et cette dissociation des registres d'inter-reconnaissance se retrouve dans les approches qui concernent la place de l'immigration algérienne dans la mémoire publique en France, qui retrouvent une trame problématique semblable, liant connaissance, interconnaissance, inter-reconnaissance.

Dans les espaces urbains et dans la vie publique, nombreuses sont les propositions historiques et mémorielles, exposées et lisibles publiquement et qui forment une sorte de vitrine composite composant ce qui peut être désigné comme une mémoire publique: des statues dédiées aux héros dans 
les espaces publics dont la toponymie est elle même souvent significative, aux décors urbains souvent porteurs de traces de mémoires collectives; des commémorations aux célébrations publiques et aux fêtes nationales inscrites dans le calendrier républicain des gestes présidentiels; à la figuration sur des scènes muséales et mémorielles, des musées et des lieux de mémoire aux animations locales. Ces propositions sont en France, en redéploiement: de nouvelles dates commémoratives viennent s'ajouter au point de saturer le calendrier commémoratif ${ }^{3}$, des rues sont parfois débaptisées et renommées, des musées d'histoire transforment leur narratif, des lieux de mémoires se transforment. La mémoire publique n'est pas inerte mais ses ambivalences actuelles à l'égard de son passé-composé (DEWITTE, 2004) placent les populations d’origine algérienne vivant en France dans une position ambiguë, et incertaine: leurs mémoires sont parfois prises en compte comme partie intégrante de la mémoire publique et de ses récits, mais, simultanément elles sont aussi considérées comme participant d'une autre mémoire nationale que celle de la France et leur histoire ne figure pas dans les récits les plus forts de la mémoire publique nationale.

D'un côté en effet l'instauration de la Cité Nationale de l'histoire de l'immigration en 2007, renommée Musée de l'histoire de l'immigration depuis le 1er janvier 2012, constitue un lieu dédié aux mémoires des migrations: mais il est lui-même ambiguë. Ce sont essentiellement l'histoire et la mémoire des migrations de travail qui constituent l'essentiel de l'exposition. Les migrations liés à la guerre d'Algérie, aux migrations coloniales sont ici lacunaires (la migration des Pieds-noirs - près d'un million de Français d'Algérie revenant en France en 1962 - n’est pas évoquée). Et hors du Musée de l’immigration - dont on a compris qu'il est loin dêtre postcolonial-, dans les autres présentations de musées d'histoire ou de société, dans les expositions de centre d'archives centrés sur l'histoire régionale, départementale ou locale, dans les lieux d'histoire et de mémoire, les migrations, et particulièrement les migrations du Maghreb ne figurent qu'au second plan, dans le cadre d'expositions temporaires. 3 Cf. KASPI, André. Rapport de la Commission de réflexion sur la modernisation des commémorations
publiques. La Documentation française, 2008. 
Par ailleurs, dans les années 2010, nombre d'actions socio-culturelles ayant figuré à l'agenda de collectivités publiques territoriales ont mis l'accent sur le thème des mémoires des immigrations, parmi lesquelles les immigrations venues du Maghreb. Ces actions sont souvent présentées par leurs promoteurs comme porteuses, implicitement ou explicitement, d'une promesse de reconnaissance publique: elles oeuvreraient à la reconnaissance « symbolique » de l'immigration en France, par la prise en compte de ses histoires et de ses récits. Mais les traductions de ces promesses en résultats restent discrètes et sont peu évaluées. Et dans les villes, se croisent, au même moment et dans des espaces différents, non seulement plusieurs types d'actions mémorielles - si ce n’est de politique mémorielle-, et plusieurs rapports à la mémoire et à l'histoire (BATTEGAY, 2008).

\section{L'IMMIGRATION ALGERIENNE DANS LA MEMOIRE PUBLIQUE NATIONALE EN FRANCE}

D’un autre côté, la colonisation (1830-1962) et la guerre d’Algérie (195462) forment en effet en France des sujets sensibles et publiquement controversés. C'est en 1999, sous la Présidence de Jacques Chirac, que la loi a reconnu qu'il s'agissait bien d'une guerre et non d' "événements " liées à des opérations effectuées en Afrique du Nord ${ }^{4}, 37$ ans après les accords d'Évian du 18 mars 1962 qui conduisent à la proclamation de l'indépendance de l’Algérie le 5 juillet de la même année. Et c’est en 2004, un demi-siècle après les début de la guerre, que des historiens publient un ouvrage sur La guerre d'Algérie, pour tenter de mettre fin à l'amnésie (HARBI; STORA, 2004), en commençant à produire une écriture historique qui ne soit pas sous l'influence du politique (BRANCHE, 2005) et de lobbies de mémoire.

Depuis lors, des initiatives officielles et des gestes présidentiels ont dessiné un paysage contradictoire. En 2005, sous la Présidence de Jacques Chirac, a été

\footnotetext{
4 Loi n. 99-882 du 18 octobre 1999 relative à la substitution, de l'expression "à la guerre d'Algérie ou aux combats en Tunisie et au Maroc" à l'expression "aux opérations effectuées en Afrique du Nord".
} 
proposée une loi mémorielle ${ }^{5}$ dont l'article 4 prescrivait que les programmes scolaires reconnaissent en particulier le rôle positif de la présence française outre-mer, notamment en Afrique du Nord. Par la suite les chefs d'Etat successifs de la France ont posé "des actes forts sur cette période de l'histoire »: en 2012 François Hollande alors Président a publié un communiqué reconnaissant «la sanglante répression » de la manifestation pacifique d'Algériens à Paris, le 17 octobre $1961^{67}$. En 2017 le Président Macron a déclaré que « la colonisation est un "crime contre l'humanité » et en 2018, il a reconnu la responsabilité de l'Etat français dans la mort de Maurice Audin.

Pour autant il n'existe en France à ce jour, en 2018, ni un Musée dédié aux relations entre la France et l'Algérie (HENRY, 2018), seulement des expositions temporaires, ni un Musée de la guerre d'Algérie selon l'expression courante de France, les Algériens la nommant comme guerre d'Indépendance. Dans les musées d'histoire et « lieux de mémoires » dédiés à l'histoire des guerres, notamment de la deuxième guerre mondiale si importante dans la reconstruction de l'identité nationale en France après la période de la collaboration et de Vichy, la guerre d’Algérie n’est presque pas évoquée. Hormis les trois colonnes du Mémorial de la guerre d'Algérie et des combats du Maroc et de la Tunisie du Quai Branly ${ }^{8}$, la guerre d’Algérie n’est pas évoquée. Le Mémorial de la prison de Montluc à Lyon, haut lieu de la mémoire nationale récemment sauvegardé de sa destruction en 2007 par le Ministère de la Défense, a été initialement dédié à la période 1943-44, au moment ou la prison était allemande, puis à la période 42-44 au moment ou elle a été la prison de Vichy: il ne fait toujours

\footnotetext{
5 Loi n ${ }^{\circ}$ 2005-158 du 23 février 2005 portant reconnaissance de la Nation et contribution nationale en faveur des Français rapatriés. L’article 4 de cette loi a été abrogé par le Décret nº2006-160 du 15 février 2006.

6 Communiqué de la présidence de la République du 17 octobre 2012 reconnaissant que "Le 17 octobre 1961, des Algériens qui manifestaient pour le droit à l’indépendance ont été tués lors d’une sanglante répression".

7 En 2016, le Président François Hollande a également institué le 19 mars (date du cessez-le-feu de la guerre d'Algérie entré en vigueur au lendemain de la signature des accords d'Évian en 1962) « journée nationale d'hommage aux "morts pour la France" pendant la guerre d'Algérie et les combats du Maroc et de la Tunisie». Cette journée avait été instituée en 2003 par le Président de la République Jacques Chirac mais fixée au 5 décembre.

8 Inauguré en 2002 par le Président de la République.
} 
pas place en 2018, dans son exposition permanente, à sa période algérienne 1958-1962, pendant laquelle 11 indépendantistes algériens ont été guillotinés sur place ${ }^{9}$. Cela relève, enquête à l'appui, non d'une ignorance ou d'un oubli mais d'un choix de non figuration, et plus précisément, d'un choix de figuration différée (BATTEGAY, 2016) comme le laissent entendre les autorités militaires l'ayant en charge.

Cette non-figuration de la Guerre d'Algérie dans les musées et les hauts lieux de la mémoire nationale résonne dans l'actualité de la société française en train de se faire, selon des lignes qui ne sont pas seulement interétatiques mais que dessinent les mémoires à l'œuvre: celles des anciens combattants français en Algérie, soldats du contingent et officiers de l'armée, des opposants français à la guerre d'Algérie, des pieds noirs (Français d'Algérie rapatriés - prés de 600 000 personnes pour la seule année 1962), des harkis également, des immigrés « algériens » de lépoque, - et des enfants de ces différents groupes pour lesquels la guerre constitue désormais une sorte de post-mémoire ${ }^{10}$. Cette mémoire de la guerre d'Algérie est imbriquée dans la société contemporaine en France: elle concerne la place faite dans la mémoire nationale, à l'histoire/mémoire des immigrés algériens et de leurs enfants, devenus en grande majorité français, dans leurs rôles d'héritiers de l'immigration.

Marquée par cinquante ans de silence public officiel, la guerre d'Algérie en France a pourtant fait l'objet de travaux d'histoire critique et d'initiatives, dans des cadres plus associatifs ou de recherches qu'institutionnels. Ces travaux d'histoire critique ont traité et traitent de fragments d'histoire: la « sanglante répression » du 17 Octobre 1961 avait été documentée par des écrits d'historiens (HOUSE; MACMASTER, 2008; EINAUDI, 2009), des témoignages, des films,

\footnotetext{
La plateforme numérique et contributive, « Montluc un lieu à mémoires multiples» (http://www.patrimonum.fr/montluc/) documente la transformation de la prison Montluc en Mémorial et donne des éléments sur la période algérienne de la prison, « oubliée » par le Mémorial actuel dédié à la période 1940-1944, et ses présentations officielles sur le site du ministère de la Défense: (http://www.memorial-montluc.fr/ et http://www.defense.gouv.fr/actualites/memoire-et-culture/le-memorial-de-la-prison-de-montluc-a-lyon. Acesso em: 30 dez. 2018. Conçu et réalisée par Marie Thérèse Tetu et Alain Battegay.

${ }^{10}$ Mohammed Harbi et Benjamin Stora (2004, p. 9) estiment ces différents groupes de mémoire à plus de cinc millions de personnes dans la France du début du XXIème siècle.
} 
des documentaires - dont certains datent de l'époque même de la guerre ${ }^{11}-$, des plateformes et des web documentaires (LE SILENCE..., 1991; LA NUIT..., 2012), en lien avec des initiatives et des associations comme Au nom de la mémoire militant pour la reconnaissance par l'Etat français de sa responsabilité dans la sanglante répression de la manifestation du 17 Octobre 1961, avant la déclaration du président Hollande ${ }^{12}$. En revanche, les massacres de Sétif et ceus de Guelma du 8 Mai 1945 qui on fait l'objet de publications d'historiens, de documentaires, et de films " grand public $»^{13}$ mais n'ont pas, à ce jour, de représentation officielle dans la mémoire nationale.

\section{LES REDEPLOIEMENTS DES « NOUS » DE LA MEMOIRE NATIONALE EN FRANCE}

L'histoire et les mémoires de la guerre d'Algérie et de la colonisation trouble l'histoire/mémoire nationale en France, mais ce n'est pas le seul redéploiement auquel cette histoire/mémoire est soumise. Dans les discours publics, les figurations muséales et les propositions d'institutions mémorielles officielles qui traitent de la mémoire nationale, la guerre de 1939-1945 est centrale: elle est présentée comme une période clef de reconstruction de l'idée nationale-étatique de la France contemporaine. Modifiant les «nous » hérités et glorieux que les musées de la Résistance mettaient en scène, et détrônant en partie le héros-résistant de son statut de seul personnage mémorable et central, la figure de la victime juive s'est imposée au cours des années 1980-90. La déportation des Juifs est en effet venue sur l'avant-scène au cours des années 80 et au début des années 90, au moment où des incitations politiques et publiques l’ont réhabilitée alors quelle avait été,

\footnotetext{
${ }^{11}$ Voir notamment les documentaires de René Vautier: Une nation, l'Algérie (1954) et L'Algerie en flammes (1958).

${ }^{12}$ Voir la chronologie de lécriture de l'histoire de la guerre France et Algérie quétablit Raphaëlle Branche (2005).

${ }_{13}$ Voir notamment les travaux de Ageron, Le Cour Grandmaison, Pervillé, Mekhaled, Peyroulou, Planche, Regui, Rey-Goldzeiguer. Voir aussi les documentaires de Adi, Hamidat, Mehdi Lallaoui et Bernard Langlois (Les massacres de Sétif - un certain 8 mai 1945), ainsi que les films Indigénes (2006) et Hors la loi (2010) du réalisateur Rachid Bouchareb, qui évoquent ces massacres.
} 
pendant plus d'un quart de siècle, masquée voire disqualifiée par la déportation des résistants, en dépit d'une historiographie disponible (HILBERG, 1988), ancienne et qui sétait depuis les années 70 fortement enrichie par des travaux portant aussi sur la collaboration et la période de Vichy faisant ressortir les zones d'ombres de la période et la responsabilité de l'Etat français dans leur déportation ${ }^{14}$. Ces travaux sont venus dans les années 90 en appui de procès Barbie en 1987 et Touvier en 1994, puis de la reconnaissance officielle et solennelle, à partir de 1995, par l'Etat français de ses crimes durant la seconde guerre mondiale avant de se traduire dans des propositions des musées et lieux de mémoires. Depuis les années 2000 la victime juive est devenue une figure centrale, en France (agrandissement du Mémorial de la Shoah et construction du mur avec les noms des 76000 déportés juifs en 2005) autant qu'au niveau international (création en 2005 d'une journée internationale dédiée à la mémoire des victimes de l'Holocauste du 27 Janvier par l'Assemblée générale des Nations Unies).

Le nous national étatique qui se recompose ici, entre histoire et mémoire, ne peut s'exonérer ni des histoires oubliées, ni des mémoires « négatives », ni des croisements de mémoires et d'histoires - selon des dimensions européennes et post-coloniales - qui le troublent aujourd'hui (LALIEU, 2015) ${ }^{15}$. Mis au défi d'articulations internes et externes problématiques, il se crispe et se redéploie par de nouveaux entrelacements entre paysages mémoriels et monstrations muséales.

\section{CE QUE LE PUBLIC FAIT AUX MEMOIRES}

Un tour réflexif semble ici en effet requis par ces redéploiements partiels des mémoires et des patrimoines à la fois pour les resituer dans les « courants

\footnotetext{
${ }^{14}$ Voir aussi les travaux de Azema, Bedarrida, Hoffman, Kaspi, Klarsfeld, Marrus, Paxton, Rousso, Sternhell. Voir aussi La politique des Justes parmi les nations, menée par Yad Vashem, et le téléfilm Shoah, de Claude Lanzmann.

${ }^{15}$ La bibliographie est abondante de la part d'historiens, d'anthropologues, de philosophes, de politistes, qui tous constatent que l'insistance sur l'entrelacement entre mémoire et histoire est venue troubler leur distinction simple, sans pour autant que les deux se confondent. Cf. les ouvrages et articles de François Hartog, Sarah Gensburger, Philippe Joutard, Reinhart Koselleck, Marie-Claire Lavabre, Paul Ricoeur, Henri Rousso, Enzo Traverso, Yosef Yerushalmi etc.
} 
de mémoires » (HALBWACHS, 1950) qu'ils forment et qui les traversent, et aussi pour restituer leurs régimes pragmatiques et les marges de manœuvre qu'ils désignent.

\section{LES ENTRELACS DES MEMOIRES COLLECTIVES ET INDIVIDUELLES ET L'EPREUVE DU PUBLIC}

Si on accepte la notion de mémoire sociale au sens de Halbwachs qui la conçoit comme englobant les mémoires individuelles et collectives à l'œuvre dans la société, on peut concevoir la mémoire publique comme un genre de filtre ou de cadrage dans la reconnaissance des mémoires, une sorte de plaque sensible qui fixe un état du public concernant les mémoires.

Les sociologues qui oeuvrent dans ce domaine se réfèrent souvent à Halbwachs, un des premiers à faire de la mémoire un objet de sociologie, pour mettre en exergue que les cadres sociaux de la mémoire sont à la fois matériels et relationnels et insister sur l'importance de l'espace comme support de mémoires. Mais les travaux de Halbwachs ne se limitent pas à mettre en vis-à-vis des groupes constitués avec des espaces de leurs mémoires, et à affirmer que « le lieu a reçu l'empreinte du groupe et réciproquement » (FORET, 2007). Au contraire. Même la notion de cadres sociaux de la mémoire proposée par Halbwachs dans son ouvrage de 1925 (HALBWACHS, 1925), au lieu d'enclore la mémoire collective dans ces vis-à-vis, l'ouvre à des témoins qui ne participent pas à la communauté relationnelle au regard de laquelle ces aspects matériels font sens (HALBWACHS, 1950). Certes Halbwachs considérait alors que la famille, la classe sociale, la religion étaient à prendre en considération comme trois grands cadres sociaux de cristallisation de la mémoire individuelle (BECKER, 2003), censés constituer autant de cadres d'appartenance des individus. Mais il insistait en même temps sur le fait que, loin d'être immuable, chacun de ces cadres fait historiquement l'objet de transformations et qu'aucun de ces cadres ne peut être tenu pour cadre exclusif d'appartenance d'individus qui font partie simultanément de plusieurs groupes de mémoire. Halbwachs soulignant ainsi 
dès cette époque que la mémoire collective est une pensée simultanément sociale et individuelle reviendra sur leurs rapports tout au long de son œuvre. Sa conception s'infléchira, comme le montre Gérard Namer (2000): de l'idée d'une mémoire collective englobant de l'extérieur les mémoires individuelles (ce qui est la thèse des Cadres sociaux...), à l'idée d'interaction continue entre mémoire individuelle et mémoire collective, " comme Simmel le disait déjà», ce qui permet de penser leur multiplicité autant que leur intériorité réciproque. Loin dêtre des touts englobant, les mémoires collectives informent les mémoires individuelles de l'intérieur, à travers les relations dans lesquelles elles se transmettent et se réinterprètent, et de l'extérieur par leurs empreintes matérielles et leurs traductions en cultures objectives et en décors (JOSEPH, 2007). Loin d'être statiques, ces mémoires collectives sont en transformations constantes, comme en témoigne l'évolution des commémorations, les transformations des musées des livres d'histoire, les modifications des marquages historiques et mémoriels, physiques et symboliques dans l'espace public. L'exposition publique des mémoires collectives et les usages publics du passé signalent ainsi, entre mémoires individuelles, mémoires collectives, mémoire sociale, l'importance des cadres publics de la mémoire.

\section{CADRES PUBLICS ET CADRES SOCIAUX DE LA MEMOIRE}

Les observations précédentes sur les redéploiements contemporains de mémoires portent autant sur des tournants de mémoire que sur des courants de mémoire, et, en tout cas, sur des mouvements de cadrage et recadrage publics des mémoires et des patrimoines. Ces mouvements de recadrage sont publics à plusieurs titres: ils impliquent des décisions et des autorités publiques, ils s'exercent publiquement en s'exposant et se rendant lisibles simultanément à plusieurs publics, leurs sens publics viennet de leur pertinence d’actualité et de tensions contemporaines entre " espace d'expériences » et « horizons d'attente » (KOSELLECK, 1990). Ils se manifestent dans les transformations de propositions 
de remémoration intentionnelle, les vitrines et propositions mémorielles opérées par les « grands entrepreneurs de mémoires » que sont l'État (de multiples manières, par des lois mémorielles ou à caractère mémoriel, par des Mémoriaux et des commémorations, par les livres scolaires...), les collectivités territoriales (par des musées, des actions culturelles, des actions sur le patrimoine, des offres de tourisme mémoriel...) ainsi que d'autres acteurs (fonctionnaires de la mémoire, milieux professionnels impliqués, certains acteurs associatifs ...). Mais les mouvements de recadrage public des mémoires se manifestent aussi dans les initiatives militantes et citoyennes d'associations, d'historiens, d'écrivains, de cinéastes, de documentaristes dans leurs rôles spécifiques. Sans oublier la part des publics dans ces transformations des paysages mémoriels et historiques: la part de spectateurs « émancipés » (RANCIÈRE, 2008), qui ne sont pas que des visiteurs captifs de musées ou de lieux dédiés à la mémoire et à l'histoire. Des spectateurs qui disposent par leur histoire-mémoire familiale, les informations auxquelles ils ont accès, leurs expériences des décors urbains et des rituels des mémoires collectives (JOSEPH, 2007), d'éléments hétérogènes par rapport aux propositions de remémoration intentionnelles et aux vitrines instituées. Ces spectateurs sont aussi des témoins de la mémoire des autres et de ce qui fait mémoire pour les autres, des témoins de la manière dont la mémoire soutenue par l'action publique fait ou non place dans ses énoncés, ses expositions, ses commémorations à des expériences socialement attestées.

Si toute une littérature, de Halbwachs à Lepetit (LEPETIT, 1993, 1999), est déjà-là pour nous dire, sans présentisme mais selon une perspective pragmatique, que la mémoire et le patrimoine se construisent et se redéfinissent au présent, dans le présent des villes et des groupes qui les composent, c'est une autre hypothèse qui semble désormais émerger. L'idée que le travail de mise en patrimoine et de mémoire au présent, en tant qu'il opère publiquement et qu'il est rendu simultanément visible à d'autres, reconfigure dans son accomplissement même des «nous » initiaux qui ne sont d'ailleurs jamais ni initiaux, ni authentiques. Au lieu d'une continuité stricte et quasi-mécanique entre cadres sociaux et cadres publics de la mémoire, c'est leur décalage et de leurs tensions qui semblent un des ressorts des redéploiements. Certes, les cadres publics formatent en partie 
la mémoire sociale, l'encadrent par ce que nous pourrions nommer, dans le langage actuel, des dispositifs d'action publique, mais ils la recadrent aussi en rendant lisibles et visibles des manières de vivre des mémoires et des histoires à des témoins qui n'en sont pas directement les acteurs et qui n'en partagent que des fragments. Au lieu de seulement s'inscrire dans le prolongement de cadres sociaux déjà donnés, les cadres et recadrages publics de la mémoire et des patrimoines les décentreraient et participeraient de leurs redéploiements.

\section{LES MEMOIRES DE L'IMMIGRATION ALGERIENNE EN FRANCE ET LES « NOUS » PUBLICS DES MEMOIRES COLLECTIVES}

Dans ces redéploiements qui entourent, impliquent, traversent les mémoires de l'immigration algérienne et de la guerre d'Algérie en France, les chemins de leur affirmation sont ainsi variés, sur plusieurs scènes de la vie publique, qu'il s'agisse des pratiques ordinaires, des récits médiatiques et des discours publics, des scènes muséales aux initiatives associatives, - chacune de ces scènes étant aussi, par moments, arènes publiques, lieux de conjonction et aussi de dispute, de controverse, de mésentente.

Cela incite à penser ce que le public fait à la mémoire, et aux mémoires des uns et des autres qui se rencontrent, s'ignorent, se croisent dans un rapport de visibilité mutuelle, donnant consistance à une figure élargie du témoin. Dans ces rapports de visibilité mutuelle, le témoin ne se confond pas avec le témoin acteur de l'histoire ou porteur de mémoire ${ }^{16}$, il est aussi témoin d'actualité, spectateur émancipé (RANCIÈRE, 2008), aujourd'hui il est aussi celui qui voit et qui juge (ARENDT, 1991) les mises en scène et les figurations publiques des mémoires collectives et les «nous publics » qu'elles promeuvent. Ce dont ce témoin peut témoigner, c'est aussi de la valeur que prennent les lieux dans les perspectives de ses contemporains, plus ou moins proches, plus ou moins autres, plus ou moins étrangers. Cette posture de « témoin de la mémoire des autres »

\footnotetext{
${ }^{16}$ Tel qu’il est identifié par les musées de la seconde guerre mondiale et de la Résistance.
} 
manque souvent à l'analyse, alors qu'à l'expérience elle est partagée par tout un chacun dans des sociétés complexes. Chacun voit les expressions publiques de la mémoire des autres autant que celle de ses groupes d'appartenances ou de références à l'occasion d'événements convoquant de la mémoire, et chacun voit lécart entre ces expressions publiques de la mémoire et leurs représentations sur des scènes dédiées (lors de cérémonies commémoratives, dans les expositions des musées et lieux de mémoire, sur les plaques commémoratives et dans le nom des rues).

Le fait que la place de l'immigration maghrébine et Algérienne soit réduite ou latérale sur ces scènes dédiées apparaît certes comme une caractéristique de la mémoire publique en France et le fait que les immigrés et leurs descendances ne soient pas des destinataires ratifiés de ces propositions de remémoration intentionnelles, signifie pour une part la place de l'immigration, notamment maghrébine, dans la perception que la société française donne d'elle-même et se donne à elle-même, en montrant ses mémoires et en racontant son histoire. Les tensions à l'œuvre entre les vitrines (ce qui est exposé) et les paysages mémoriels (ceux qui sont vus à partir de regards multi-orientés) seront-elles reconnues en France comme un des défis et une des sources des redéploiements de l'offre actuelle de remémoration intentionnelle?

Dans ces conditions, aucune promesse de reconnaissance simple ne peut être soutenue (WALZER, 1997) qui laisserait supposer que la monstration des mémoires de l’immigration Algérienne aurait un effet de reconnaissance publique, "par en haut », qui serait bénéfique à ceux qui les portent, - sauf à renouer avec une posture affirmant le bien pensant des mémoires urbaines et se retrouvant à l'épreuve du présent des villes. La promesse implicite de reconnaissance faite aux immigrés ne peut se limiter à la seule perspective «verticale» de reconnaissance. La seule promesse soutenable serait, qu'en passant au filtre du public, les mémoires de l'immigration gagnent en visibilité autant qu'en consistance historique ou en connaissances localisées, et, que, partie prenante encore latérale dans les compositions actuelles d'un paysage mémoriel en mouvements, elles inscrivent sur la place publique devenue membrane, au delà de la compassion ou de la culpabilité, des configurations d'avenir. 


\section{CONCLUSION: LES VULNERABILITES DE L'INTER- RECONNAISSANCE DANS LE FAIRE SOCIETE}

Ce regard rétrospectif plaide ainsi pour des philosophies du politique plutôt que des philosophies politiques, compatibles avec les perspectives et les travaux d'anthropologie urbaine et politique qui nous montrent qu'on ne passe pas aussi facilement de la ville à la cité, du citadin au citoyen, en superposant espaces publics et espaces communs, reconnaissance et inter-reconnaissance (BATTEGAY, 2000).

En portant l'attention sur des expériences des populations d’origine Algérienne en France, sur des moments d'affirmation publique d'un «faire place » et d'un «prendre place » qui se manifestent dans des compositions urbaines et s’éprouvent dans les arènes controversées de la mémoire publique, ces approches convergent pour considérer que les épreuves de reconnaissance, en matière de droit de cité ne se satisfont pas de la figure du citoyen, comme sujet de droit, et de sa capacité juridique et politique à intervenir dans les affaires de la cité.

L'exploration des dimensions du vivre-ensemble impliquées dans ces expériences montre que l’appartenance à la nationalité ne suffit pas à comprendre les grammaires ${ }^{17} \mathrm{du}$ faire place et du prendre place, qui se lisent dans les compositions urbaines et les manière de vivre les villes, autant que dans les troubles de la mémoire publique et les normes de conjonction qu'elle légitime entre mémoires collectives.

Cela incite à se retourner vers les problématiques de la reconnaissance: en confirmant d'abord, à l'aune de ces expériences, que la méconnaissance n'est pas la seule source de non-reconnaissance, même si d'une certaine manière elle l'entretient, comme le montrent les difficultés à penser l'ethnicité en France; en inquiétant aussi les conceptions paisibles des sociabilités faisant des relations d'interconnaissance et de bon voisinage des sortes de garanties d'inter-reconnaissance. Les troubles de reconnaissance de l'autre en tant que co-sociétaire ne jouent pas ici sur fond d'ignorance mutuelle, ni de déficit de liens

\footnotetext{
17 Voir les travaux du Groupe de Sociologie Politique et Morale, autour de Laurent Thévenot.
} 
d'interconnaissance, ils font au contraire apparaitre les liens d'interconnaissance et de voisinage comme dispositifs vulnérables de mitoyenneté et d'hospitalité, y compris dans les rapports de citoyenneté.

Les modalités d'inter-reconnaissance, ambiguës et ambivalentes, qui s'observent, autant d'en haut que du dedans, dans des compositions et des agencements urbains, ainsi que dans les tensions qui traversent la mémoire sociale, entre mémoires collectives et mémoire publique interrogent les épreuves et les compétences politiques du vivre ensemble, sans concevoir l'inter-reconnaissance comme le résultat de jeux infinis de négociations et en manque d'ultimité (RICOEUR, 2002), mais comme opérant aussi selon des registres dissociés et en interaction.

De telles démarches socio-anthropologiques privilégient les aspects analytiques plus que normatifs des problématiques de la reconnaissance. D’une part, elles incitent à des enquêtes et à des observations ethnographiques et de sociographiques non-normatives répondant à un intérêt politique d'analyse et de connaissance du vivre-ensemble, et de ses épreuves. D’autre part, elles croisent dans leurs mouvements, les problématiques du pluralisme que travaillent les philosophies qui se tiennent aux bords du politique. Ancrées dans la caverne d'expériences sociales avec lesquelles elles tentent de penser, elles inquiètent les catégories qui organisent notre compréhension des sociabilités et du faire société, en manifestant non seulement des troubles de reconnaissance et des formes de mépris, mais les vulnérabilités des dispositifs d’inter-reconnaissance.

\section{RÉFERENCES}

1. ARENDT, Hannah. Juger: sur la philosophie politique de Kant. Paris: Seuil, 1991.

2. BATTEGAY, Alain. Les Beurs dans l'espace public. Esprit, v. 6, n.102, p. 113-119, 1985.

3. BATTEGAY, Alain. Approches urbaines de l'immigration: la question des territoires ethniques dans les villes françaises. Revue Européenne des Migrations Internationales, v. 8, n. 2, p. 83-100, 1992. 
4. BATTEGAY, Alain. L'espace commun, entre mythe et reconstructions: variations. In: METRAL, Jean. (coord.). Cultures en ville, ou de l'art et du citadin. La Tour d'Aigues: Éditions de l’Aube, 2000. p. 241-254.

5. BATTEGAY, Alain. Les recompositions d'une centralité commerçante immigrée: la Place du Pont à Lyon. Revue Européenne des Migrations Internationales, v. 19, n. 2, p. 9-22, 2003.

6. BATTEGAY, Alain. Localité, commercialité, ethnicité. La Place du Pont à Lyon et Dubaï. Approche contrastive et entrecroisée de deux carrefours urbains d'échanges et de migrations. In: Audebert, Cédric; Ma Mung, Emmanuel. (ed.). Les nouveaux territoires migratoires: entre logiques globales et dynamiques locales, Bilbao: Université de Deusto, 2007. p. 153-171.

7. BATTEGAY, Alain. Les redéploiements des nous du patrimoine et de la mémoire en France. Cultures et Recherches, n. 114-115, p. 28-30, 2008. Disponível em: http://bit.ly/2J6WJin. Acesso em: 7 fev. 2018.

8. BATTEGAY, Alain. The transformation of Montluc Prison (Lyon) into a Memorial, an Outstanding National Memory Space, and « Forgetting » the Prison's Algerian Period. In: GUIXÉ I COROMINES, Jordi. (ed.). Past and power: public policies on memory. Debates, from global to local. Barcelona: Edicions de la Universitat de Barcelona, 2016. p. 188-203.

9. Becker, Annette. Maurice Halbwachs: un intellectuel en guerres mondiales 1914-1945. Paris: Viénot, 2003.

10. BEKKAL, Kadidja. Des êtres en négatif: les jeunes de familles algériennes en France. Paris: CIEM, 1981.

11. BORDREUIL, Samuel. Politique de la reconnaissance, politiques de la sociologie. In: PAYET, Jean Paul; BATTEGAY, Alain. La reconnaissance à l'épreuve: explorations socio-antropologiques. Lilles : Presses du Septentrion, 2008. p. 73-81.

12. Isaac Joseph: la politique depuis Goffman. In: CEFAÏ, Daniel; SATURNO, Carole. (coord.). Itinéraires d'un pragmatiste: autour d'Isaac Joseph. Paris: Economica, 2007. p.101-134.

13. Boubeker, Ahmed. Les mondes de l'ethnicité: la communauté d’expérience des héritiers de l’immigration maghrébine. Paris: Éditions Balland, 2003.

14. BRANCHE, Raphaëlle. La guerre d'Algérie une histoire apaisée ? Paris: Seuil, 2005.

15. DEWITTE, Philippe. Immigration et intégration: l'état des savoirs. Paris: La Découverte, 1999.

16. 16. DEWITTE, Philippe. La France se conjugue au passé composé. Hommes et Migrations, n. 1247, p. 1-4, 2004. 
17. EINAUDI, Jean Luc. Scènes de la guerre d'Algérie en France: automne 1961. Paris: Le Cherche midi, 2009.

18. Foret, Catherine. (coord.).Travail de mémoire et requalification urbaine: repères pour l'action. JOUVE, 2007. Disponível em: http://bit.ly/2Tokecq. Acesso em: 7 fev. 2018 .

19. GOFFMAN, Erwin. L'ordre de l'interaction: les moments et leurs hommes. Paris: Minuit, 1988.

20. GUILLON, Michèle; TABOADA-LEONETTI, Isabelle. Le triangle de Choisy: un quartier chinois à Paris. Paris: L'Harmattan-Ciemi, 1986.

21. Halbwachs, Maurice. Les cadres sociaux de la mémoire. Paris: Albin Michel, 1925.

22. Halbwachs, Maurice. La mémoire collective. Paris: Albin Michel, 1950.

23. HARBI, Mohammed; STORA, Benjamin. La guerre d'Algérie: la fin de l'amnésie (1954-2004). Paris: Robert Laffond, 2004.

24. HENRY, Jean-Robert. L'histoire aux prises avec les mémoires: l'exemple du musée avorté de Montpellier sur l'histoire de la France et de l'Algérie. L'année du Maghreb, v.19, p. 133-164, 2018.

25. Hilberg, Raoul. La destruction des juifs d'Europe. Paris: Fayard, 1988.

26. HOUSE, Jim; MACMASTER, Neil. Paris 1961: les Algériens, la terreur d'Etat et la mémoire. Paris: Tallandier, 2008.

27. JOSEPH, Isaac. Pluralisme et contiguïtés. In: CEFAÏ, Daniel; JOSEPH, Isaac. (ed.). L'héritage du pragmatisme: conflits d'urbanité et épreuves de civisme. La Tour d'Aigues: Editions de l'Aube, 2002. p. 83-105.

28. JOSEPH, Isaac. Rituels et décor de la mémoire collective, d'après Maurice Halbwachs. In: L'athlète moral et l'enquêteur modeste: recueil d'articles. Paris: Economica, Études sociologiques, 2007. p. 295-305.

29. KOSELLECK, Reinhart. Le futur passé: contribution à la sémantique des temps historiques. Paris: Edition de l'École des Hautes Études en Sciences Sociales, 1990.

30. Lalieu, Olivier. Histoire de la mémoire de la Shoah. Saint-Cloud: Soteca, 2015.

31. LA NUIT oubliée 17 Octobre 1961. Direção: LAMBERT, Olivier; SALVA, Thomas. [S. l.]: Hans Lucas, 2011. Web documentário (15 min). Disponível em: https:// lemde.fr/2VRr8mk. Acesso em: 7 fev. 2018.

32. Lepetit, Bernard. Le présent de l'histoire. In: Lepetit, Bernard. (coord.). Les formes de l'experience: une autre histoire sociale. Paris: Albin Michel, 1993. p. 273-298.

33. Lepetit, Bernard. Carnet de croquis: sur la connaissance historique. Paris: Albin Michel, 1999. 
34. LE SILENCE du fleuve. Direção: DENIS, Agnès; LALLAOUI, Mehdi. [S. l.]: Raspouteam, 1991. Web documentário (52 min). Disponível em: http://bit. ly/2CesFLR. Acesso em: 7 fev. 2018.

35. MA MUNG, Emmanuel. Territorialisation marchande et négociation des identités: les Chinois de Paris. Espace et Société, n. 96, p. 145-162, 1999.

36. Martinello, Marco. L'ethnicité dans les sciences sociales contemporaines. Paris: PUF, 1995.

37. Namer, Gérard. Halbwachs et la mémoire sociale. In: DÉLOYE, Yves; HAROCHE, Claudine. Maurice Halbwachs: espaces, mémoire et psychologie collective. Paris: Éditions de la Sorbonne, 2000. p. 107-113.

38. NICOLAS, Guy. Fait ethnique et usage du concept d'ethnie. Cahiers Internationaux de Sociologie, v. 14, p. 95-126, 1973.

39. PAYET, Jean Paul; BATTEGAY, Alain. La reconnaissance à l'épreuve: explorations socio-anthropologiques. Lille: Presses du Septentrion, 2008.

40. RANCIÈRE, Jacques. Le spectateur émancipé. Paris: La Fabrique Editions, 2008

41. RAULIN, Anne. Espaces marchands et concentrations minoritaires: la Petite Asie de Paris. Cahiers Internationaux de Sociologie, v. 85, p. 225-241, 1988.

42. RAULIN, Anne. Mise en scène des commerces maghrébins parisiens. Terrain, n. 7, p. 24-33, 1986.

43. RAULIN, Anne. L'ethnique est quotidien: diasporas, marchés et cultures métropolitaines. Paris: L’harmattan, 2000.

44. Ricoeur, Paul. La critique et la conviction. Paris: Seuil, 2002.

45. SAYAD, Abdelmalek. Les enfants illégitimes. Actes de la Recherche en Sciences Sociales, n. 25, p. 61-81, 1979.

46. Simon, Patrick; Stavo-Debauge, Johan. Les politiques anti-discrimination et les statistiques: paramètres d'une incohérence. Sociétés Contemporaines, n. 53, p. 57-84, 2004.

47. TARRIUS, Alain. Le foncier à deux vitesses in Etudes foncières, 1989/L’entrée dans la ville: migrations maghrébines et recomposition des tissus urbains à Tunis et à Marseille. Revue Européenne des Migrations Internationales, v. 3, p. 131-148, 1987.

48. TARRIUS, Alain. Identités en mouvement: sédentarités, nomadisme et recompositions urbaines. Peuples Méditerranéens, n. 43, p. 87-104, 1988.

49. TARRIUS, Alain. Les fourmis de l'Europe. Paris: INRETS, 1990. 
50. TOTO, Francesco; Pénigaud de Mourgues, Théophile; Renault, Emmanuel. La reconnaissance avant la reconnaissance: archéologie d'une problématique moderne. Lyon: ENS Editions, 2017.

51. TOUBON, Jean-Claude; MESSAMAH, Khelifa. Centralité immigrée: le quartier de la Goutte d'Or. Paris: L'Harmattan: Ciemi, 1991.

52. TUOT, Thierry. La grande nation: pour une société inclusive. Rapport au Premier ministre sur la refondation des politiques d'intégration. La documentation française, 2013. Disponível em: http://bit.ly/2EWqcGo. Acesso em: 7 fev. 2018.

53. WALZER, Michael. Interpretation and social criticism. Cambridge: Harvard University Press, 1987.

54. WALZER, Michael. Sphères de justice: une deféfense du pluralisme et de l'égalité. Paris: Seuil, 1997. 